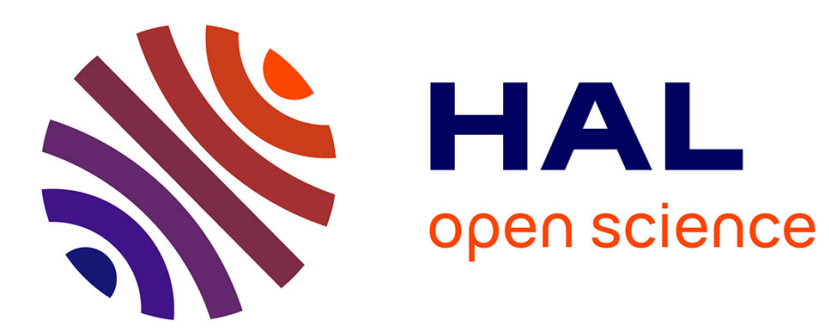

\title{
NOUVELLES ÉTUDES SUR LE DOSAGE DES MATIÈRES MINÉRALES DES CASÉINES LACTIQUES
}

\author{
Jean Pien, M. Weissmann
}

\section{- To cite this version:}

Jean Pien, M. Weissmann. NOUVELLES ÉTUDES SUR LE DOSAGE DES MATIÈRES MINÉRALES DES CASÉINES LACTIQUES. Le Lait, 1939, 19 (181), pp.15-22. hal-00927781

\section{HAL Id: hal-00927781 \\ https://hal.science/hal-00927781}

Submitted on 1 Jan 1939

HAL is a multi-disciplinary open access archive for the deposit and dissemination of scientific research documents, whether they are published or not. The documents may come from teaching and research institutions in France or abroad, or from public or private research centers.
L'archive ouverte pluridisciplinaire HAL, est destinée au dépôt et à la diffusion de documents scientifiques de niveau recherche, publiés ou non, émanant des établissements d'enseignement et de recherche français ou étrangers, des laboratoires publics ou privés. 
bouteilles a fait diminuer lès frais d'exploitation et que le lait présenté et mis à la disposition du client sous cette forme a occasionné également une augmentation de la consommation très profitable au développement de l'industrie laitière. Dans cet ordre d'idées, nous rappellerons ici les conseils d'Henry FORD :

"Faites que les marchandises soient aussi bonnes et aussi bon marché que possible. Evitez le gaspillage. Que tout le travail qui peut être exécuté par une machine soit fait à la machine et non à la main. Lorsque le consommateur est satisfait de Ja qualité de la marchandise livrée, la vente augmente, les frais généraux diminuent et le producteur est assuré d'obtenir un bénéfice intéressant. »

\section{NOUVELLES ÉTUDES SUR LE DOSAGE DES MATIÈRES MINÉRALES DES CASÉINES LACTIQUES}

par

\section{JEAN PIEN}

Ingénieur Chimiste (I. C. R.), Docteur ès Sciences,

Directeur des Laboratoires des Fèrmiers Réunis.

\author{
M. WEISSMANN \\ Chimiste \\ des Laboratoires \\ des Fermiers Réunis.
}

Dans une étude antérieure (Le Lait, novembre 1933, p. 1081), l'un de nous a montré que le dosage des matières minérales des caséines lactiques par la méthode ancienne de calcination pure et simple, conduisait à des résultats erronés pour les deux raisons suivantes :

10 Il y a, au cours de la calcination, fixation d'une partie du phosphore or zanique qui ne devrait pas être compté.

$2^{\circ}$ Il subsiste dans les cendres des matières organiques incomplètement brûlées.

Ces deux causes agissent dans le même sens pour fausser par excès le dosage des matières minérales.

Le remède proposé pour redresser cette situation était le suivant :

"Incorporer à la caséine avant calcination une certaine quantité connue de matières minérales dont le but est de fixer tout le phosphore organique et de permettre l'obtention de cendres blanches exemptes de résidus organiques incomplètement brûlés. Retrancher du résultat l'excès de matières minérales introduites ainsi que le taux constant de phosphore organique ainsi fixé (en $\mathrm{P}^{2} \mathrm{O}^{5}: 1,6 \%$ de la caséine). ” 
Le sel minéral utilisé était l'acétate de calcium - solution à 30 grammes de $\mathrm{CaO}$ par litre environ - dont on employait $5 \mathrm{~cm}^{3}$ pour 5 grammes de caséine.

Plusieurs années de pratique quotidienne de cette méthode nous ont confirmés dans l'excellence du principe, mais nous ont appris qu'il y avait lieu de modifier le détail opératoire.

Le but de la présente note est d'exposer et de justifier cette modification.

$$
\text { *** }
$$

\section{Inconvénients de la chaux}

La méthode préconisée en 1933 prescrivait donc l'emploi, pour 5 grammes de caséine, de $5 \mathrm{~cm}^{3}$ d'une solution d'acétate de calcium à 30 grammes de $\mathrm{CaO}$ par litre. Les cendres de 5 grammes de caséine (de l'ordre de 50 milligrammes) étaient done surchargées d'un poids de 150 milligrammes de chaux qu'il fallait ensuite retrancher.

Cette opération ne correspond à aucune impossibilité pratique. Mais la chaux que l'on vient de calciner est très hygroscopique et reprend du poids par hydratation avec une très grande rapidité même si l'on prend soin d'utiliser un dessiccateur à anhydride phosphorique. Le fait d'ouvrir ce dessiceateur pour y introduire la capsule fait entrer dans l'appareil une certaine quantité d'humidité qui est partiellement absorbée par la chaux en même temps que par l'anhydride. Le temps nécessaire à la pesée permet ensuite une nouvelle reprise d'humidité inévitable et variable. Sur une quantité de chaux aussi importante que 150 milligrammes, ces reprises peuvent influencer sérieusement le résultat.

Nous avons done songé tout d'abord à utiliser des solutions d'acétate de chaux moins concentrées : à 20 ou à 10 grammes de $\mathrm{CaO}$ par litre. La reprise de poids en un temps donné et pour un état hygrométrique donné de l'atmosphère est, en effet, d'autant plus faible que le poids de chaux introduit est lui-même moins élevé.

En dosant les matières minérales d'une caséine aveo diverses solutions d'acétate de chaux, nous avons trouvé (après avoir retranché le taux de chaux introduit) :

\begin{tabular}{|c|c|c|}
\hline $\begin{array}{c}\text { Concentration en } \mathrm{CaO} \text { des solutions } \\
\text { d'acétate }\end{array}$ & $\begin{array}{c}\text { Cendres }\left(\mathrm{P}^{2} \mathrm{O}^{5} \text { non }\right. \\
\text { déduit) }\end{array}$ & Cendres ( $\mathrm{P}^{2} \mathrm{O}^{5}$ déduit) \\
\hline 10 grammes par litre.. & 2,17 & 0,57 \\
\hline 20 grammes par litre ....... & 2,43 & 0,83 \\
\hline 30 grammes par litre .......... & 2,47 & 0,87 \\
\hline
\end{tabular}


Les accroissements de poids sont évidemment proportionnels aux quantités de chaux employées.

Il est donc certain qu'avec de petites quantités on atténue l'erreur; mais on ne la supprime pas. D'ailleurs, on ne peut pas descendre au-dessous d'une certaine quantité de chaux : avec la solution d'acétate à 5 grammes de $\mathrm{CaO}$ par litre, on ne minéralise pas complètement la caséine; les cendres obtenues ne sont pas blanches.

Un autre facteur intervient : le temps qui s'écoule entre la sortie du mecker et la fin de la pesée. Nous avons constaté que, même dans le dessiccateur à $\mathrm{P}^{2} \mathrm{O}^{5}$, la reprise de poids est proportionnelle au temps de séjour.

En effet :

\begin{tabular}{|c|c|c|c|}
\hline \multirow{2}{*}{$\begin{array}{c}\text { Concentration en } \mathrm{CaO} \text { des solutions } \\
\text { d'acétate }\end{array}$} & \multicolumn{2}{|c|}{$\begin{array}{c}\text { Cendres } \%\left(\mathrm{P}^{2} \mathrm{O}^{5} \text { non déduit }\right) \\
\text { après un séjour } \\
\text { dans le dessiccateur de }\end{array}$} & \multirow{2}{*}{ Ecarts } \\
\hline & 10 minutes & 15 minutes & \\
\hline 10 grammes par litre $\ldots \ldots \ldots \ldots$ & 2,17 & 2,21 & 0,04 \\
\hline 20 grammes par litre $\ldots \ldots \ldots \ldots$ & 2,43 & 2,59 & 0,16 \\
\hline 30 grammes par litre $\ldots \ldots \ldots \ldots$ & 2,47 & 2,67 & 0,20 \\
\hline
\end{tabular}

L'erreur due au temps de séjour dans le dessiccateur est d'autant plus importante que la quantité de chaux mise en œuvre est plus élevée. Même pour les solutions les moins riches elle n'est pas négligeable.

La conclusion de ces observations, répétées à de nombreuses reprises, est donc la suivante : l'emploi de l'acétate de chaux comme minéralisant introduit dans la détermination des cendres une erreur par excès qui peut être importante.

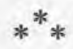

\section{Avantages de la magnésie}

Il convenait donc de faire appel à un sel fournissant par calcination un oxyde très peu hygroscopique. Nous avons choisi l'acétate de magnésium.

Les inconvénients signalés pour la chaux ne sont pas rigoureusement supprimés, mais ils sont réduits à des proportions insignifiantes que l'on peut négliger, En effet : 
10 Influence de la concentration des solutions d'acétate de MIg

\begin{tabular}{|c|c|c|}
\hline & \multicolumn{2}{|c|}{ Cendres $\left(\mathrm{P}^{2} \mathrm{O}^{5}\right.$ non déduit) } \\
\hline & Caséine 1 & Caséine 2 \\
\hline Solution à 16 grammes de $\mathrm{MgO}$ p. L. & 2,20 & 1,76 \\
\hline Solution à 32 grammes de $\mathrm{MgO}$ p. L. . & 2,24 & 1,80 \\
\hline Ecarts $\ldots \ldots \ldots \ldots \ldots \ldots \ldots \ldots$ & 0,04 & 0,04 \\
\hline
\end{tabular}

$2^{\circ}$ Influence du temps de séjoup dans le dessiccateur.

\begin{tabular}{c|c|c}
\hline \hline 10 minutes & 60 minutes & Ecarts \\
\cline { 2 - 3 } 1,76 & 1,79 & 0,03 \\
1,80 & 1,84 & 0,04 \\
\hline
\end{tabular}

Si maintenant nous mettons en parallèle des dosages effectués par les deux méthodes sur diverses caséines lactiques, nous constaterons avec netteté l'importance des erreurs que l'on a pu commettre par l'emploi de la chaux :

\begin{tabular}{|c|c|c|c|c|c|c|}
\hline \multirow[b]{2}{*}{ Avec $\mathrm{MgO} \ldots \ldots \ldots \ldots \ldots$} & \multicolumn{6}{|c|}{ Cendres de diverses easéines ( $\mathrm{P}^{2} \mathrm{O}^{5}$ non déduit) } \\
\hline & 1,80 & 1,93 & 2,08 & 2,20 & 2,02 & 2,15 \\
\hline Avec $\mathrm{CaO} \ldots \ldots \ldots \ldots \ldots$ & 1,88 & 1,98 & 2,22 & 2,29 & 2,18 & 2,20 \\
\hline $\begin{array}{l}\text { Erreur en trop due à la chaux, } \\
\text { en } \% \text { des résultats de la ma- } \\
\text { gnésie } \quad \ldots \ldots \ldots \ldots \ldots \ldots \ldots \ldots \ldots\end{array}$ & $4,4 \%$ & $2,5 \%$ & $6,7 \%$ & $4,1 \%$ & $8,0 \%$ & $2,3 \%$ \\
\hline
\end{tabular}

Ces erreurs pourraient paraître minimes. Mais les résultats exprimés ci-dessus s'entendent $\mathrm{P}^{2} \mathrm{O}^{5}$ non déduit. Si on retranche comme convenu $1,6 \%$ au taux de cendres - correspondant à $\mathrm{P}^{2} \mathrm{O}^{5}$ organique fixé, considéré comme constant - les erreurs dues à la chaux prennent une importance relative plus sérieuse :

\begin{tabular}{|c|c|c|c|c|c|c|c|}
\hline \multirow{2}{*}{ Avec $\mathrm{MgO}$} & \multirow{2}{*}{$\frac{}{\ldots \ldots \ldots}$} & \multicolumn{6}{|c|}{ Cendres de diverses caséines $\left(\mathrm{P}^{2} \mathrm{O}^{5}\right.$ déduit $)$} \\
\hline & & 0,20 & 0,33 & 0,48 & 0,60 & 0,42 & 0,55 \\
\hline Avec $\mathrm{CaO}$ & & 0,28 & 0,38 & 0,62 & 0,69 & 0,58 & 0,60 \\
\hline
\end{tabular}


Empressons-nous de signaler que les dosages de matières minérales en présence de chaux, effectués dans notre laboratoire, l'ont été avec un soin extrême (dessiccateurs à $\mathrm{P}^{2} \mathrm{O}^{5}$ de petits volumes, temps de séjour au dessiccateur exactement contrôlé, pesées très rapides, etc.) parce que nous étions prévenus des erreurs possibles.

Aussi les écarts que la méthode à la chaux peut provoquer entre les mains d'un expérimentateur non averti de cette circonstance particulière, sont-ils toujours beaucoup plus importants que ceux que nous avons relatés, alors que la méthode à la magnésie n'exige pas de préeautions aussi sévères et ne risque pas d'entraîner de graves erreurs.

$$
\text { *** }
$$

\section{II. Nouvelle technique proposée}

5 grammes de caséine finement broyée sont mélangés dans une capsule de quartz ou de platine à $5 \mathrm{~cm}^{3}$ d'une solution d'acétate de magnésium cristallisé à environ 100 grammes par litre, correspondant à 15-20 grammes de $M g O$ par litre, exactement titrée par calcination (1). On laisse gonfler pendant une demi-heure pour que la caséine s'imprègne parfaitement du sel de magnésium. On sèche à l'étuve et on calcine, modérément d'abord, pour finir à température élevée (mecker).

On porte au dessiccateur à anhydride phosphorique pendant 10 minutes et on pèse.

Connaissant le titre en $\mathrm{MgO}$ de la solution magnésienne, on corrige les cendres de la quantité de base introduite.

\section{Expression des résultats.}

Si le résultat est exprimé tel quel, il est indispensable de l'accompagner de la mention " $\mathrm{P}^{2} \mathrm{O}^{5}$ organique non déduit" puisque le chiffre obtenu comprend tout le phosphore organique fixé par la magnésie.

Si l'on retranche le terme fixe 1,60 , correspondant au $\mathrm{P}^{2} \mathrm{O}^{5}$ du phosphore organique - considéré comme constant, à 0,1 près $\%$ de caséine - il convient d'ajouter au résultat la mention " $\mathrm{P}^{2} \mathrm{O}^{5}$ organique déduit $"$.

Ces deux manières de présenter le résultat sont également admissibles. La première est peut-être plus rigoureuse. La seconde fait intervenir l'incertitude qui peut régner sur le taux réel du phosphore organique des caséines. Dans les caséines très pauvres en

(1) La solution d'acétate de $\mathrm{Mg}$ à 100 grammes par litre exactement, correspond à $18 \mathrm{gr}$. 6 de $\mathrm{N}$ gO (acétate à 4 molécules d'eau). La conservation de ces solutions d'acétate de magnésium s'obtient aisément en les saturant de chloroforme (quelques centimètres cubes par litre) ou en les additionnant d'une trace de phénol. 
cendres, cette incertitude peut entraîner une inexactitude importante en valeur relative.

Et, comme nous l'avons montré dans notre précédent travail sur ce sujet, il ne saurait être question de doser réellement le $\mathrm{P}^{2} \mathrm{O}^{5}$ de la caséine pour le déduire du résultat, car on risque ainsi de retrancher du phosphore minéral qui doit figurer dans le taux de cendres.

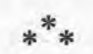

\section{Impossibilité d'utiliser les cendres sulfuriques}

Dans certaines industries, pour des matières dont les cendres sont difficiles à exécuter correctement, on détermine les "cendres sulfuriques ». Pour cela on ajoute à la matière quelques gouttes d'acide sulfurique. On chasse l'excès d'acide à température modérée puis on calcine. Les cendres "réelles» sont obtenues en faisant usage d'un coefficient empirique de transformation.

1. Cette manière de faire, qui aurait pu simplifier le dosage des cendres des caséines lactiques, ne saurait être admise pour les raisons suivantes :

$1^{\circ}$ La calcination des cendres en présence d'acide sulfurique, entraîne une perte importante et variable suivant la quantité d'acide sulfurique employé et suivant la durée de l'opération.

Voici par exemple ce que nous avons obtenu sur des cendres traitées par l'ancienne méthode à la chaux avant et après traitement à l'acide sulfurique :

$\begin{array}{cc}\begin{array}{c}\text { Avant le traitement } \\ \text { sulfurique }\end{array} & \begin{array}{c}\text { Après le traitement } \\ \text { sulfurique (1) }\end{array} \\ 2,12 & 1,86 \\ 2,22 & 2,10 \\ 2,29 & 1,98 \\ 2,18 & 2,02 \\ 2,17 & 1,90 \\ 2,25 & 1,86 \\ 2,17 & 1,74 \\ 2,19 & 1,92 \\ 2,17 & 1,90 \\ 2,17 & 1,40\end{array}$

$2^{\circ}$ Nous avońs pensé que cette perte devait être due à une disparition partielle de l'acide phosphorique de la caséine.

Il en est bien ainsi. En effet :
$\mathrm{P}^{2} \mathrm{O}^{5}$ total sur la caséine initiale (attaque
sulfo-nitrique) $\ldots \ldots \ldots \ldots \ldots \ldots \ldots \ldots$
1,71
1,68
(1) Correction faite des $\mathrm{SO}^{\prime}$ fixés sur la ehaux. 
$\mathrm{P}^{2} \mathrm{O}^{5}$ dosé sur le résidu de la calcination sul-

$\begin{array}{rll}\text { furique } \ldots \ldots \ldots \ldots \ldots \ldots \ldots \ldots \ldots \ldots \ldots \ldots & 1,03 & 0,78 \\ \text { Perte en } \mathrm{P}^{2} \mathrm{O}^{5} \% \text { de la caséine } \ldots \ldots \ldots \ldots \ldots \ldots & 0,68 & 0,90 \\ \text { Perte de } P^{2} O^{5} \% \text { du } P^{2} O^{5} \text { initial } \ldots \ldots \ldots \ldots \ldots & 39 \% & 53 \%\end{array}$

Cette perte ne correspond évidemment pas à la totalité du $\mathrm{P}^{2} \mathrm{O}^{5}$ organique (qui vaut environ $1,60 \%$ de la caséine) et dont une partie a pu se fixer pendant la calcination malgré la présence de l'acide sulfurique. Peut-être plusieurs traitements sulfuriques répétés parviendraient-ils à éliminer tout le phosphore organique. Mais la méthode se compliquerait et on risquerait d'ailleurs de commettre une autre erreur en libérant à son tour une partie du $\mathrm{P}^{2} \mathrm{O}^{5}$ minéral de la caséine qui serait perdu à la calcination finale.

$3^{\circ}$ On démontre en effet cette perte de phosphore minéral dans la calcination sulfurique en opérant de la manière suivante : on fixe d'abord tout le phosphore organique par la chaux ou la magnésie en appliquant la méthode générale indiquée plus haut; on dose le phosphore total sur ces cendres, ce qui donne l'acide phosphorique total de la caséine; on traite par l'acide sulfurique des cendres analogues obtenues en présence de chaux et on y dose le phosphore après calcination sulfurique. Or, on constate une perte très importante de $\mathrm{P}^{2} \mathrm{O}^{5}$.

En effet :

\begin{tabular}{|c|c|c|}
\hline chaux $\ldots \ldots \ldots \ldots \ldots \ldots \ldots \ldots \ldots$ & 1,66 & 1,62 \\
\hline $\begin{array}{r}\mathrm{P}^{2} \mathrm{O}^{5} \text { sur ces mêmes cendres traitées à l'acide } \\
\text { sulfurique } \ldots \ldots \ldots \ldots \ldots \ldots \ldots \ldots \ldots \ldots \ldots \ldots \ldots \ldots\end{array}$ & 1,18 & 0,99 \\
\hline Perte en $\%$ de la caséine ............ & 0,48 & 0,63 \\
\hline Perte de $P^{2} O^{5} \%$ du $P^{2} O^{5}$ initial & $29 \%$ & $38 \%$ \\
\hline
\end{tabular}

L'acide sulfurique ne pouvant chasser le phosphore organique qui est déjà minéralisé par le traitement à la chaux ou à la magnésie, ne peut que faire perdre ce phosphore minéralisé lui-même.

En d'autres termes, si cette hypothèse se confirnie, il faut admettre que la calcination sulfurique est capable de faire perdre une partie de l'acide phosphorique du phosphate tricalcique.

$4^{\circ}$ Or, nous pouvons donner une démonstration directe de ce fait important :

Calcinons un mélange de phosphate tricalcique et d'acide sulfurique jusqu'à disparition complète de l'acide sulfurique; reprenons le résidu par $\mathrm{HCl}$ en prenant toutes les précautions nécessaires pour éviter de laisser dans la capsule on dans l'insoluble $\left(\mathrm{SO}^{4} \mathrm{Ca}\right)$ du phosphate tricalcique qui échapperait au dosage dans le filtrat. Enfin, dosons l'acide phosphorique dans le résidu soigneusement repris, lavé, filtré, etc. 
On constate une perte importante de $\mathrm{P}^{2} \mathrm{O}^{5}$ variant dans nos essais de 12 à $22 \%$ de l'acide phosphorique du phosphate tricalcique mis en œuvre. La perte croît avec la quantité d'acide sulfurique utilisé.

Notre hypotbèse se trouve donc vérifiée.

C'est la condamnation de la technique des cendres sulfuriques, au moins dans les produits contenant de l'acide phosphorique soit organique, soit même combiné à une base terreuse.

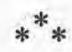

\section{Conclusion}

La meilleure méthode pour la détermination exacte des matières minérales de la caséine lactique nous paraît être celle que nous avons antérieurement décrite (Le Lait, novembre 1933, p. 1081), mais à la condition de remplacer la solution d'acétate de calcium par une solution d'acétate de magnésium.

\section{LES PHOSPHATIDES DANS LE RÉSIDU DU BEURRE}

\section{par le Dr BRuno REWALD}

de Londres

La proportion de phosphatides dans le beurre n'est pas très élevée; mais la présence de ces phosphatides est importante, parce que nous savons qu'elles ont une influence nette sur les propriétés de cette matière grasse si appréciée. Dans quelques pays du Continent, surtout en Suisse, on utilise un procédé spécial pour séparer la matière grasse pure du beurre de tous les autres composants normaux du beurre : eau, caséine, sucre de lait, sel, etc. Ce procédé consiste à chauffer la matière grasse du beurre à une température telle que toute l'eau s'évapore. Cette opération effectuée, les autres sous-produits deviennent absolument insolubles; toute la structure du "beurre" est modifiée, l'émulsion est détruite et les substances non huileuses se séparent au fond. La matière grasse du beurre claire et exempte d'eau se conserve très longtemps sans se détériorer si on la conserve en boîtes de fer-blanc ou autres récipients.

Le résidu consiste en un produit brun sombre qui ressemble à de la graisse et qui contient naturellement toutes les "impuretés " du beurre ci-dessus mentionnées, mais contient aussi encore une certaine proportion de matière grasse du beurre et, en outre, la plupart des phosphatides qui sont séparées et précipitées. La température élevée à laquelle ce résidu est obtenu a naturellement aussi une influence sur la composition de ses différents composants, mais 